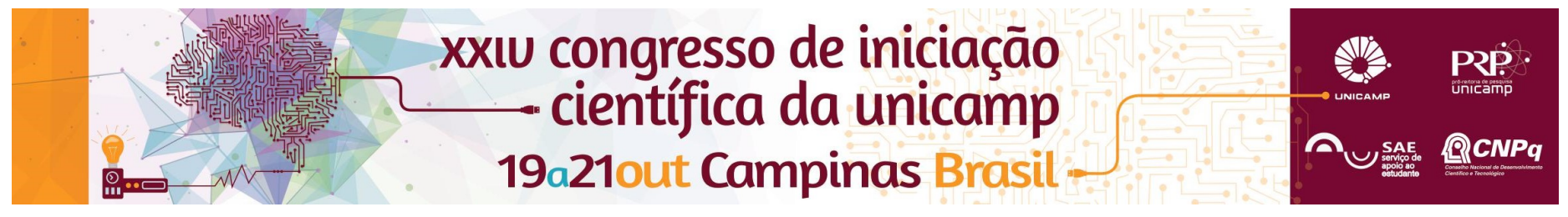

\title{
Introduction to Particle Physics and a Study of Jet Production at the LHC
}

\author{
Gabriella Dantas Franco*, Jun Takahashi.
}

\begin{abstract}
Particle Jets are widely seen structures in LHC high energy events, which are generally studied by Jet algorithms such as FastJet. The main focus of the project was to study Jet structures generated in 7 TeV proton-proton collisions by analysing simulated events generated by Monte Carlo code PYTHIA. To do so, we used two particle angular correlation method, which generally is used to search for collective behavior of systems.
\end{abstract}

\section{Key words:}

Particle Jets, LHC, Correlation.

\section{Introduction}

The Large Hadron Collider (LHC) from CERN is today the world's largest particle accelerator, that can generate highest energy particle collisions up to $13 \mathrm{TeV}$ in the center of mass.

At such energies, when partons (the constituents of particles) scatter they generate collimated particle structures that form a cone, the Particle Jets. Due to being widely present in LHC events, understanding better their behavior is really important to comprehend fundamental processes such as particle production, which is not yet completely understood.

To study the production of such structures, we used an analysis technique known as two particle angular correlation.

The use of FastJet ${ }^{1}$, an specific jet finding algorithm, is very common in the study of Jets, because it helps to define and find important parameters for such structures.

In this work our aim was to do a detailed two particle angular correlation study in Particle Jets, and compare with the same study done with FastJet parameters. Also we wanted to understand how changing the frame of reference and chemical initial composition changes the final Jet observables.

\section{Results and Discussion} steps.

The development of this work was done in three major

The first one consisted in using the Monte-Carlo based particle collision event generator PYTHIA ${ }^{2}$ to create simulated Jet events. These events were constructed simulating the decay of the Z0 particle into two quarks, which would then fragment into two particle Jets. These two jet events were the main object of our study. In the simulation code, it was possible to chose the flavor of the quark pair into which the Z0 would decay, hence allowing us to study also possible dependencies of the Jet structure in the chemical composition of the original fragmenting quark.

The second one consisted in the development of the two particle angular correlation analysis tool, which was made in $\mathrm{C}++$ language using the ROOT platform.

The third step was seeking for an observable that could be used to see the Jet behavior in the analysis. So it was chosen the Jet width, as calculated from the standard deviation:

$$
\sigma=\sqrt{\frac{1}{N} \sum_{i=1}^{N}\left(x_{i}-\bar{x}\right)^{2}}
$$

in which $\bar{x}$ is the average of the values.

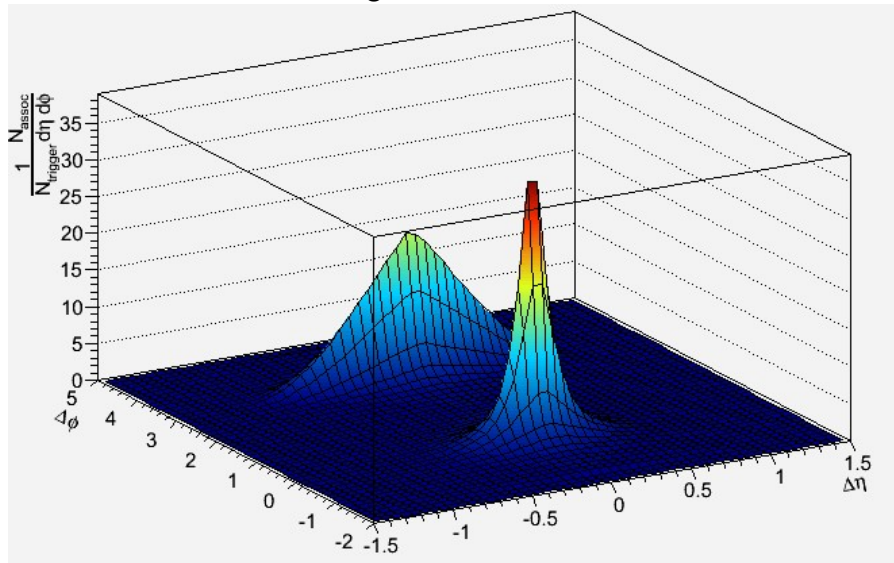

Image 1: Image from two particle angular correlation analysis showing the Jet structures.

\section{Conclusions}

It was noticed from this work that the two particle angular correlation study in simulated LHC events allow the visualization of Particle Jet structures, despite being a simple analysis method.

It was observed that this parameter (the width) can change with the analysis. For example, the choice of frame of reference in which the analysis is done can change drastically the Jet width measured. The change of reference from the decaying particle frame to the laboratory frame generates a widening of the Jet width. Another important parameter is the reference angle to which the two particle correlation is applied. Changing from the Jet direction to the direction of the leading particle of the Jet also generates a widening of the observed width.

We also studied the variation of the Jet width with the quark flavor. Some small variation was observed, but a more detailed and quantitative analysis is still ongoing.

\section{Acknowledgement}

The authors thank the support received from CNPq for the development of this project.

${ }^{1}$ FastJet User Manual, arXiv:1111.6097

${ }^{2}$ PYTHIA 6.4 Physics and Manual, arXiv:hep-ph/0603175

${ }^{3}$ ROOT, https://root.cern.ch 